\title{
Quantification and Representation of Structural Heterogeneities in a Continuum Model of the Mouse Heart
}

\author{
J. Rao*, M. Ellisman**, H. Hakozaki**, R. Price***, J. H. Omens* and A. D. \\ McCulloch* \\ * Department of Bioengineering, University of California, San Diego, La Jolla, CA \\ 92093-0419 \\ **Center for Research in Biological Sciences, University of California, San Diego, La \\ Jolla, CA 92093-0608 \\ ***Cell and Developmental Biology and Anatomy. University of South Carolina, \\ Columbia, SC 29208
}

With the availability of genetically engineered mice models of heart disease, there is a need for more structurally detailed computational models of murine ventricular anatomy suitable for predictive simulations of physiological function. In the heart, gap junctions (GJ) provide low resistance pathways, enabling coordinated propagation of action potential between cells. A decrease of GJ coupling causes a slowing of conduction, which is a key ingredient of reentrant arrhythmias [2]. In order to study the regional variations in structure and cell connectivity in detail, the purpose of the study was to measure and quantify the heterogeneities of GJ distribution and statistically represent them in a finite element (FE) model of the mouse heart.

Epicardial and endocardial contours of 20-micron frozen serial sections of 3-4 month old NIH Swiss mice hearts were fitted in prolate spherical coordinates to construct the FE model of the heart anatomy. Each heart was sectioned along approximately orthogonal planes (short-axis, anterior-posterior and left-right). Approximately 10,000 cleavage orientations per heart were measured automatically and used to reconstruct regional fiber and laminar sheet orientations.

To extend the model to account for regionally varying anisotropic electrical conductivity, frozen sections of another heart were immunolabled with anti-Cx43 and either F-actin or wheat germ agglutinin (WGA) and imaged with a 2-photon confocal microscope using either a 20x or a 40x lens. High-intensity signal from immunoflurescence (IF) images of Cx43 (Figure 1A) was separated from background to quantify the transmural variations in GJs . The relative Cx43 quantity was defined as the proportion of total myocardial tissue area occupied by the IF signal of $\mathrm{Cx} 43$ [3]. In order to get a statistical representation of patterns of cell connectivity, a cell of interest was picked in a 3-D volume stack of images as seen in figure 2. Distribution of cells about the cell of interest was calculated by demarcating its boundaries and the boundaries of the surrounding cells and ensuring that GJ plaques connect them [4]. Distribution was measured about the centroid of the cell of interest.

In this study both transmural as well as local variations of GJ distribution in the heart were measured. Our preliminary results show a lower concentration of $\mathrm{Cx} 43$ at subepicardium and increased levels in the mid-myocardium and endocardium (Figure 1B). 
These heterogeneities can be correlated to transmural heterogeneities in conduction velocity [1]. By measuring local variations, we get an indication of the number and direction of connections between cells, which can be correlated to longitudinal vs. transverse conduction velocity. Both these quantities can be parameterized as diffusion coefficient of electrical propagation in the FE model. Future work will involve using this model to investigate functional effects of these heterogeneities in the whole heart.

\section{References}

1. S. Poelzing et. al. Am. J. Phisiol. Heart Circ Physiol 286 (2004) H2001.

2. S. Rohr et. al., Circulation Research. 83 (1198) 781.

3. J. Saffitz et. al., Am. J. Physiol Heart Circ Physiol. 278 (2000) H1662.

4. R. Luke et. al., J. Clin. Invest. 87 (1991) 1594.

5. This research was supported by NIH through the National Biomedical Computation Resource (P41 RR08605) and NSF grant (BES-0086482).

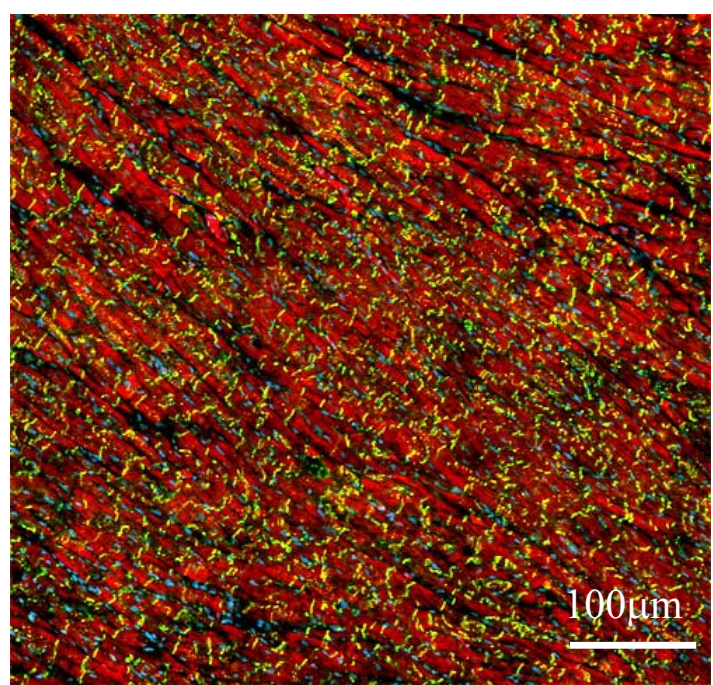

Figure 1A: Ventricular tissue labeled with antiCx43 (green), F-actin (red) and nuclei (blue)

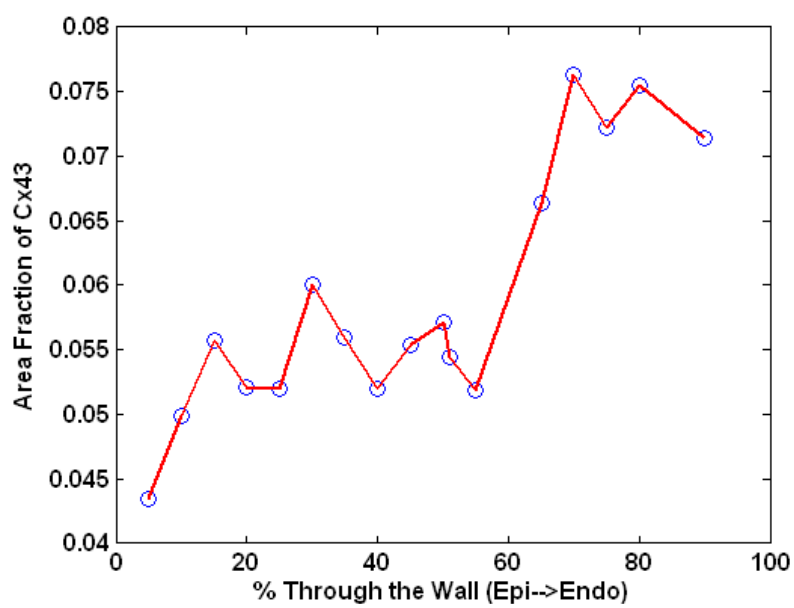

Figure 1B: Transmural variation in $\mathrm{Cx} 43$

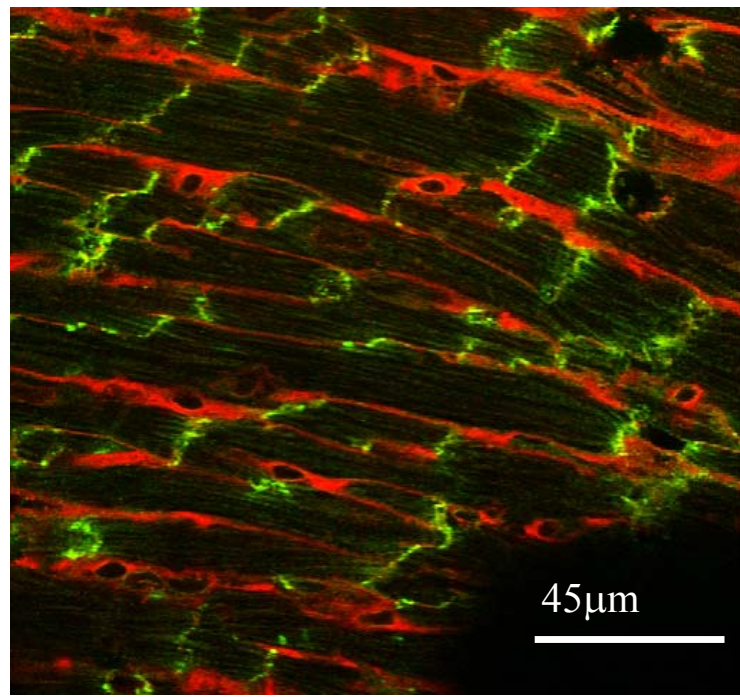

Figure 2: Ventricular tissue labeled with WGA (red) and anti-Cx43 (green) 